\title{
SERIAL RINGS WITH KRULL DIMENSION
}

\author{
by A. W. CHATTERS
}

(Received 30 September, 1988)

1. Introduction. A module is said to be serial if it has a unique chain of submodules, and a ring is serial if it is a direct sum of serial right ideals and a direct sum of serial left ideals. The serial rings of Krull dimension 0 are the Artinian serial (or generalised uniserial) rings studied by Nakayama and for which there is an extensive theory (see for example [4]). Warfield in [10] extended the theory to the non-Artinian case. In particular he showed that a Noetherian serial ring is a direct sum of Artinian serial rings and prime Noetherian serial rings, and he gave a structure theorem in the prime Noetherian case. A Noetherian non-Artinian serial ring has Krull dimension 1. Serial rings of arbitrary Krull dimension have been studied by Wright ([9], [12], [13], [14]) with special results being proved when the Krull dimension is 1 or 2 .

In this paper, we extend some of these results to serial rings of arbitrary Krull dimension. The methods used rely heavily on making use of weak chain conditions. In Section 3, it is shown that certain indecomposable serial rings with Krull dimension are prime or can be written as triangular matrices, extending results of Singh, Warfield and Wright. A structure theorem for prime serial rings of finite Krull dimension is given in Section 4, which generalises results of Warfield and Wright when the Krull dimension is 1 or 2.

2. Background material and conventions. All rings considered here are associative with identity element. We shall use $N$ or $N(R)$ to denote the nil radical of a ring $R$, and $J$ or $J(R)$ to denote the Jacobson radical of $R$. The set of elements regular modulo an ideal $I$ will be denoted by $C(I)$. We refer to [3] and [5] for general material on ring theory and Krull dimension.

Let $R$ be a ring. As in [10], an $R$-module $M$ is said to be serial if for any submodules $A$ and $B$ of $M$ we have $A \subseteq B$ or $B \subseteq A$. The ring $R$ is said to be serial if it is the direct sum of serial right ideals and is also the direct sum of serial left ideals. Such rings are discussed in [10] and Chapter 6 of [3]. Serial rings with Krull dimension have been studied in [9], [12], [13], [14]. It is shown in Theorem 6 of [14] that the left and right Krull dimensions of a serial ring are equal. A serial ring $R$ is Noetherian if and only if $\cap J^{n}=0$, and under these conditions $\operatorname{Kdim}(R) \leqslant 1$ [10, Theorem 5.11].

Let $R$ be a serial ring with Krull dimension. Then $N$ is nilpotent by Theorem 5 of [6]. Also $R / N$ is a serial semi-prime Goldie ring [5, Theorem 5.1] in which every finitely-generated one-sided ideal is projective [10, Theorem 4.1]. Therefore $R / N$ is a direct sum of prime rings by Theorem 4.3 of [7]. Also $R$ has an Artinian quotient ring if and only if $R$ satisfies the ascending chain condition for right annihilators [11, Theorem 5].

Let $R$ be an indecomposable non-singular serial ring. Then $R$ has a two-sided quotient ring which is a blocked triangular matrix ring over a division ring $D[10$, Theorem 4.1]. The corresponding full matrix ring over $D$ is both the maximal left and right quotient ring of $R$. Hence every complement one-sided ideal of $R$ is generated by an idempotent [1, Theorems 5.2 and 2.4]. The structure of $R$ can now be described

Glasgow Math. J. 32 (1990) 71-78. 
completely in terms of blocked triangular matrix rings over prime rings as in Theorem 6.10 of [1]. We shall use this in Corollary 4.3 to give the structure of non-singular serial rings of finite Krull dimension.

3. Certain serial rings are prime. We shall establish some sufficient conditions for a serial ring with Krull dimension to be prime. The first result of this type was Warfield's theorem that an indecomposable Noetherian non-Artinian serial ring $R$ is prime (in this case $\operatorname{Kdim}(R)=1$ ). A result of this type when $\operatorname{Kdim}(R)=2$ was proved by Wright in Proposition 2.7 of [12].

Lemma 3.1. Let $R$ be a serial ring with Krull dimension and let $K$ be a non-zero serial right $R$-module with $K N=0$. Then there is a unique minimal prime ideal $P$ of $R$ such that $K P=0$. Also $K Q=K$ for every minimal prime ideal $Q$ of $R$ with $Q \neq P$.

Proof. We know that $K N=0$ and that $N$ contains a product of minimal primes (because $R / N$ is a Goldie ring). Also $K \neq 0$. Therefore there is a minimal prime ideal $P$ with $K P \neq K$. Let $Q$ be a minimal prime ideal of $R$ with $Q \neq P$. Because $R / N$ is a direct sum of prime rings, we have $P+Q=R$. Hence $K=K P+K Q$. But $K P \neq K$ and $K$ is serial. Therefore $K=K Q$. Let $Q_{1}, \ldots, Q_{n}, P$ be the distinct minimal primes of $R$. Then $K Q_{i}=K$ for all $i$ and $Q_{1} Q_{2} \ldots Q_{n} P \subseteq N$. Therefore $0=K N=K Q_{1} \ldots Q_{n} P=K P$.

Lemma 3.2. Let $R$ be a serial ring and let $K$ be a semi-prime ideal of $R$ such that $R / K$ is a Goldie ring and $K$ contains no non-zero idempotent elements of $R$. Then $K=K c=c K$ for all $c \in C(K)$.

Proof. This is based on Remark (6) at the end of Chapter 6 of [3]. Let $c \in C(K)$. By Theorem 3.3 of [10], there are non-zero orthogonal idempotents $e_{1}, \ldots, e_{n}$ of $R$ adding to 1 such that each $e_{i} R$ is a serial right $R$-module and $c R=\sum_{i}\left(e_{i} R \cap c R\right)$. We fix an integer $j$ with $1 \leqslant j \leqslant n$. We have $e_{j} \notin K$. Also $(c R+K) / K$ is an essential right ideal of $R / K$. Hence $e_{j} R \cap(c R+K)$ is not contained in $K$. Because $e_{j} R$ is serial, it follows that $e_{j} R \cap(c R+K) \supsetneq e_{j} K$. But

$$
e_{j} R \cap(c R+K)=e_{j} R \cap\left(\sum_{i}\left(e_{i} R \cap c R\right)+\sum_{i} e_{i} K\right)=e_{j} R \cap c R+e_{j} K
$$

Hence $e_{j} R \cap c R+e_{j} K \supsetneqq e_{j} K$. Because $e_{j} R$ is serial, it follows that $e_{j} K \subseteq e_{j} R \cap c R$. Hence $e_{j} K \subseteq c R$ for all $j$ and $e_{1}+\ldots+e_{n}=1$. Therefore $K \subseteq c R$. Because $c \in C(K)$, we have $c R \cap K=c K$. Therefore $K=c K$.

ThEOREM 3.3. Let $R$ be an indecomposable serial ring with Krull dimension and suppose that $R$ has a non-nilpotent ideal $X$ such that $\bigcap_{n=1}^{\infty} X^{n}=0$. Then $R$ is a prime ring.

Proof. We fix a minimal prime ideal $P$ of $R$ such that $P$ does not contain $X$. We wish to show that $N=N P$. Let $e$ be an idempotent element of $R$ such that $e R$ is serial. It is enough to show that $e N=e N P$, and this is trivial if $e N=0$. Suppose that $e N \neq 0$ and that $e N \neq e N P$. By taking $K=e N / e N^{2}$ in Lemma 3.1, we see that $e N P=e N^{2}$. Because $X$ is not contained in $P$, there is no minimal prime of $R$ which contains $X+P$. Therefore 
$c \in X+P$ for some $c \in C(N)$. But $N c=N$ by Lemma 3.2. Hence

$$
e N=e N c=e N(X+P)=e N X+e N P=e N X+e N^{2} .
$$

Therefore $e N=e N X=e N X^{2}=\ldots$ This is a contradiction because $e N \neq 0$ and $\cap X^{n}=0$.

Thus $N=N P$. Because $R / N$ is a direct sum of prime rings, we know that $P / N$ is generated by a central idempotent of $R / N$. Hence there is an idempotent element $f$ of $R$ such that $P=f R+N=R f+N$. Thus

$$
N=N P=N f+N^{2}=N f+N\left(N f+N^{2}\right)=N f+N^{3}=\ldots
$$

Hence $N=N f$ and $P=R f+N=R f+N f=R f$. By symmetry, we have $P=R f=f R$. It follows that $f$ is a central idempotent element of $R$. Therefore $P=0$ because $R$ is indecomposable.

COROLLARY 3.4 (Warfield). Let $R$ be a serial ring. Then $R /\left(\bigcap_{n=1}^{\infty} J^{n}\right)$ is a direct sum of
(Wian rings and prime rings. Artinian rings and prime rings.

Proof. Without loss of generality, we may suppose that $\cap J^{n}=0$ and that $R$ is indecomposable. Either $J$ is nilpotent and $R$ is Artinian, or $J$ is not nilpotent and $R$ is prime by Theorem 3.3 .

Corollary 3.5 (Wright [12, Proposition 2.7]). Let $R$ be an indecomposable serial ring with Krull dimension. Set $J_{1}=\bigcap_{n=1}^{\infty} J^{n}$. Suppose that $J_{1}$ is not nilpotent and that $\bigcap_{n=1}^{\infty} J_{1}^{n}=0$. Then $R$ is a prime ring.

TheоRем 3.6. Let $R$ be an indecomposable serial ring with Krull dimension. Suppose that $R$ has an Artinian quotient ring and that $\mathrm{Kdim}(N)<\mathrm{Kdim}(R)$ as right $R$-modules. Then there is a unique minimal prime ideal $P$ of $R$ such that $\operatorname{Kdim}(R / P)=\operatorname{Kdim}(R)$. Also $P=R f$ for some idempotent element $f$ of $R$.

Proof. Because $\mathrm{Kdim}(R / N)=\mathrm{K} \operatorname{dim}(R)$, there is a minimal prime $P$ of $R$ such that $\operatorname{Kdim}(R / P)=\mathrm{Kdim}(R)$. We shall show that $N=N P$. Suppose that $e$ is an idempotent element of $R$ such that $e N$ is a non-zero serial right $R$-module and $e N P=e N^{2}$; we shall obtain a contradiction. We have $\operatorname{Kdim}(e N / e N P) \leqslant \operatorname{Kdim}(N)<\operatorname{Kdim}(R)=\operatorname{Kdim}(R / P)$. Let $x$ be a non-zero element of $e N / e N P$. Then $\mathrm{Kdim}(x R)<\mathrm{Kdim}(R / P)$. Therefore $x R$ is torsion as a right $R / P$-module. Hence $x c=0$ for some $c \in C(P)$. Let $y \in e N$ with $y+e N P=x$. Then $y(c R+P) \subseteq e N^{2}$. But $d \in c R+P$ for some $d \in C(N)$. Thus $y d \in e N^{2}$. But $N=N d$ by Lemma 3.2 or [11, Theorem 5]. Thus $y d \in e N^{2} d$. Because $R$ has an Artinian quotient ring, we know that $d$ is a regular element of $R$. Therefore $y \in e N^{2}$, i.e. $x=0$. This is a contradiction. It follows, as in the proof of Theorem 3.3, that $N=N P$ and that $P=R f$ for some idempotent $f$.

Suppose that $Q$ is a minimal prime of $R$ with $Q \neq P$ and $\operatorname{Kdim}(R / Q)=\operatorname{Kdim}(R)$. Then $N=N Q$ and $Q=R g$ for some idempotent $g$. We have $g \notin P$, i.e. $g R \neq g P$. By Lemma 3.1 with $K=g R / g N$, we have $g N=g P$. Thus $Q P=R g P=R g N \subseteq N$. Hence $N^{2} \supseteq N Q P=N P=N$. Therefore $N=0$. But $R$ is indecomposable. Therefore $R$ is prime. This is a contradiction because $P \neq Q$.

COROLlaRY 3.7. Let $R$ be an indecomposable serial ring with Krull dimension. Suppose that $R$ has an Artinian quotient ring and that $\operatorname{Kdim}(N)<\operatorname{Kdim}(R)$ as both left $R$-modules and right $R$-modules. Then $R$ is a prime ring. 
CoRollary 3.8 (Singh [8, Theorem 2.11]). Let $R$ be an indecomposable serial right Noetherian ring which is neither prime nor right Artinian. Then

$$
R \cong\left[\begin{array}{cc}
S & M \\
0 & T
\end{array}\right]
$$

where $S$ is a prime Noetherian serial ring, $T$ is an Artinian serial ring, and $M$ is an $S$-T-bimodule.

Proof. We know that $R$ has an Artinian quotient ring ([11, Theorem 5] or [3, Theorem 6.10]). Also $N c=N$ for every regular element $c$ of $R$ (Lemma 3.2 or [11, Theorem 5]). But $N$ is finitely-generated as a right ideal. Therefore $N$ is Artinian as a right $\boldsymbol{R}$-module (see for example the proofs of [2, Lemma (A)] or [3, Lemma 5.2]). Thus $\operatorname{Kdim}(N)=0$ and $\operatorname{Kdim}(R)=1$ as right $R$-modules. Let $P=R f$ as in Theorem 3.6. Then

$$
R \cong\left[\begin{array}{cc}
S & M \\
0 & T
\end{array}\right]
$$

where $S \cong R / P$ and $T \cong f R f$. The minimal primes of $T$ correspond to minimal primes $Q$ of $R$ with $Q \neq P$. But $R / Q$ is Artinian for every such $Q$ by Theorem 3.6. It follows that $T$ is Artinian.

4. Prime serial rings of finite Krull dimension. We shall determine the structure of prime serial rings with finite Krull dimension in terms of blocked matrices over integral domains. The cases in which the Krull dimension is 1 or 2 were done by Warfield [10, Theorem 5.14] and Wright [12, Theorem 2.11]. The proof will be module-theoretic and will require that the domain of definition of certain homomorphisms can be extended. The following lemma gives enough injectivity for this purpose and was suggested by Theorem 1.4 of [4].

LEMMA 4.1. Let $R$ be a serial ring with primitive idempotents $e$ and $f$. Let $x \in e R$, and let $a: x R \rightarrow f R$ be a homomorphism of right $R$-modules. Then one at least of the following statements is true.

(1) There is a homomorphism $b: e R \rightarrow f R$ with $b(x)=a(x)$, or

(2) there is a homomorphism $b: f R \rightarrow e R$ with $b a(x)=x$.

Proof. There are orthogonal primitive idempotents $g_{1}, \ldots, g_{n}$ of $R$ adding to 1 such that each $R g_{i}$ is a serial left $R$-module. We have $x R=x g_{1} R+\ldots+x g_{n} R$. Because $e R$ is serial, we have $x R=x g_{i} R$ for some $i$. Thus $x R=x g R$ for some primitive idempotent $g$. Set $w=a(x g)=a(x) g$. Because $R g$ is serial, we have $R x g \subseteq R w$ or $R w \subseteq R x g$.

Case (a). Suppose that $R x g \subseteq R w$. Then $x g=r w$ for some $r \in R$. But $x=e x$ and $w=f w$. Hence, without loss of generality, we may suppose that $r=e r f$. Define $b: f R \rightarrow e R$ by $b(f s)=r s$ for all $s \in R$. Then $b a(x g)=b(w)=r w=x g$. But $x R=x g R$. Hence $x=x g t$ for some $t \in R$. Therefore $b a(x)=b a(x g t)=(b a(x g)) t=x g t=x$.

Case (b). Suppose that $R w \subseteq R x g$. Then $w=r x g$ for some $r \in R$ with $r=f r e$. Define $b: e R \rightarrow f R$ by $b(e s)=r s$ for all $s \in R$. Then $b(x g)=r x g=w=a(x g)$. As in (a), it follows that $b(x)=a(x)$.

THEOREM 4.2. Let $R$ be a prime serial ring with finite Krull dimension. Then there is a serial integral domain $T$ such that either

(1) $R \cong M_{n}(T)$ for some positive integer $n$, or 
(2) there is a positive integer $k \neq 1$ and sets $H_{i j}$ for $1 \leqslant i, j \leqslant k$ such that $R$ is isomorphic to the ring of $k$ by $k$ matrices with $(i, j)$-entries in $H_{i j}$, where

(i) if $i<j$ then $H_{i j}$ is the set of all $n_{i}$ by $n_{j}$ matrices with entries in $T$ for some positive integers $n_{i}$ and $n_{j}$,

(ii) if $i>j$ then $H_{i j}$ is the set of all $n_{i}$ by $n_{i}$ matrices over $J(T)$, and

(iii) each $H_{i i}$ is a prime serial ring of finite Krull dimension and with smaller Goldie rank than that of $R$.

Thus each $H_{i i}$ can in turn be written as a matrix ring in the same way that $R$ is, and so on.

Proof. We shall frequently use the well-known fact that a nonzero homomorphism between uniform right ideals of $R$ is injective. Set $J=J(R)$. Suppose firstly that $J$ is a prime ideal of $R$. Then all simple right $R$-modules are isomorphic. There are orthogonal idempotents $e_{1}, \ldots, e_{n}$ of $R$ adding to 1 such that each $e_{i} R$ is a serial right $R$-module. Each $e_{i} R / e_{i} J$ is a simple module. Therefore $e_{i} R / e_{i} J \cong e_{j} R / e_{j} J$ for all $i$ and $j$. It is now routine to show that $e_{i} R \cong e_{j} R$ and that (1) holds with $T=\operatorname{End}_{R}\left(e_{1} R\right)$.

For the remainder of the proof, we suppose that $J$ is not a prime ideal of $R$. Set $J_{0}=J$ and $J_{n}=\bigcap_{i=1}^{\infty} J_{n-1}^{i}$ for every positive integer $n$. Because $R$ has finite Krull dimension, we have $J_{h}=0$ for some $h$ [14, Theorem 6]. For each $i$, let $K_{i}$ be the ideal of $R$ containing $J_{i}$ such that $K_{i} / J_{i}=N\left(R / J_{i}\right)$. Each $K_{i}$ contains no non-zero idempotent element of $R$. We have $K_{0}=J$ and $K_{h}=0$. Thus $K_{0}$ is not prime and $K_{h}$ is prime. Let $p$ be the largest integer such that $K_{p}$ is not a prime ideal. Set $K=K_{p}$ and $L=K_{p+1}$. These meanings for $K$ and $L$ will be retained for the rest of the proof.

Set $I=\bigcap_{i=1}^{\infty} K^{i}$ and let $e$ be a primitive idempotent of $R$. We shall show that $I=L$. Because $K$ is nilpotent modulo $J_{p}$ we have $I=\bigcap_{i=1}^{\infty} K_{p}^{i}=\bigcap J_{p}^{i}=J_{p+1} \subseteq L$. Also $L^{r} \subseteq J_{p+1}$ for some non-negative integer $r$. Hence $L^{r} \subseteq J_{p} \subseteq K$. Therefore $L \subseteq K$. But $L$ is prime and $K$ is not. Therefore $L \subsetneq K$. Let $i$ be a positive integer. Then $e K^{i}$ is not contained in the prime ideal $L$. Because $e R$ is serial it follows that $e K^{i} \supsetneq e L$. Because 1 is the sum of such idempotents $e$, we have $L \varsubsetneqq K^{i}$. Hence $L \subseteq I$. Therefore $L=I=J_{p+1}$. From " $e K^{i} \supsetneq(\geqslant L$ ", it also follows that $e K^{i} \neq e K^{j}$ if $i \neq j$.

The reason for choosing $K$ to be semi-prime but not prime is that we take $k$ in (2) to be the number of prime ideals of $R$ minimal over $K$.

Set $C=C(K)$. We shall show that $C$ is an Ore set in $R$. Let $a \in R$ and $c \in C$. Because $R / K$ is a semi-prime Goldie ring we have $a d=c b+u$ for some $d \in C, b \in R, u \in K$. By Lemma 3.2, we have $u=c v$ for some $v \in K$. Thus $a d=c(b+v)$. Therefore $C$ is an Ore set in $R$, and the elements of $C$ are regular in $R$. Let $S$ be the partial quotient ring of $R$ with respect to $C$. Then $K=J(S)$ and $L$ is a prime ideal of $S$. Because $\cap K^{i}=L$ it follows that $S / L$ is a prime Noetherian serial ring.

Let $e$ be a primitive idempotent of $R$. Then $e R$ is a serial $R$-module. Hence $e S$ is serial as a right $S$-module. For each $i$, set $M_{i}=\operatorname{ann}_{S}\left(e K^{i} / e K^{i+1}\right)$. Because $S / L$ is a prime Noetherian serial ring and $e K^{i} \supseteq e L$ for all $i$, it follows, as in the proof of [10, Theorem 5.14], that the sequence $M_{0}, M_{1}, M_{2}, \ldots$ starts by running through the distinct maximal ideals of $S$ and then repeats itself. The only way in which $e$ influences this sequence is in determining its starting point. In $R$, this means that: the primes of $R$ minimal over $K$ are 
precisely the ideals of the form $\operatorname{ann}_{R}\left(e K^{i} / e K^{i+1}\right)$ for some non-negative integer $i$; $\operatorname{ann}_{R}\left(e K^{i} / e K^{i+1}\right)=\operatorname{ann}_{R}\left(e K^{j} / e K^{j+1}\right)$ if and only if $i \equiv j \bmod (k)$, where $k$ is the number of primes of $R$ minimal over $K$; ann $\left(e K^{i} / e K^{i+1}\right)$ is determined by ann $\left(e K^{i-1} / e K^{i}\right)$ and not by $e$.

Notation. From now on, $g$ denotes a fixed primitive idempotent element of $R ; P$ is a fixed prime ideal of $R$ minimal over $K$ such that $g R \neq g P$, i.e. $g K=g P$ (Lemma 3.1); $T=\operatorname{End}_{R}(g K)$.

We shall show that $T \cong \operatorname{End}_{s}(g S)$. Recall that $K=J(S)$. Let $a \in T$. For $x \in g K$ and $c \in C(K)$, we have $a\left(x c^{-1}\right) c=a\left(x c^{-1} c\right)=a(x)$, i.e. $a\left(x c^{-1}\right)=a(x) c^{-1}$. Thus $a: g K \rightarrow g K$ is a right $S$-module homomorphism. Because $g S / g L$ is a cyclic serial module over the Noetherian ring $S / L$, we have $g K=y S$ for some $y \in S$. We shall use Lemma 4.1 to show that $a$ can be extended to an element of $\operatorname{End}_{S}(g S)$. Suppose that there is a right $S$-module homomorphism $b: g S \rightarrow g S$ such that $b(y)=y$. Then $b(g K) \supseteq b(a(g K))=b a(y S)=y S=$ $g K$. Hence $b(g K)=g K$ and $b(g S)=g S$. Thus $b$ is an automorphism of $g S$ and its inverse is an extension of $a$. If no such $b$ exists then $a$ can be extended to an element of End ${ }_{s}(g S)$ by Lemma 4.1 . It follows that $T \cong \operatorname{End}_{s}(g K) \cong \operatorname{End}_{s}(g S)$.

From now on, we identify $T$ with $\operatorname{End}_{S}(g S)$. We have $J(T)=\{a \in T: a(g S) \subseteq g K\}$. Let $a \in T$ with $a \neq 0$. Then $a(g S) / a(g K) \cong g S / g K$, and $g S / g K \cong g K^{i} / g K^{i+1}$ if and only if $i$ is divisible by $k$. Hence $J(T)=\left\{a \in T: a(g S) \subseteq g K^{k}\right\}=\left\{a \in T: a(g K) \subseteq g K^{k+1}\right\}$.

Let $e$ be a primitive idempotent element of $R$. Because $L$ is prime, we have $e R g \nsubseteq L=\bigcap_{i=1}^{\infty} K^{i}$. Let $i$ be the smallest positive integer such that $e R g \nsubseteq K^{i}$, i.e. such that there is a right $R$-module homomorphism $u: g R \rightarrow e R$ with $u(g R)$ ? $e K^{i}$. These meanings for $i$ and $u$ will be fixed until further notice. We have $e K^{i-1} \supseteq u(g R) \supsetneq e K^{i}$. Hence $u(g P)=u(g K) \subseteq e K^{i}$. Let $Q$ be any prime of $R$ minimal over $K$ with $Q \neq P$. Then $P+Q=R$ and $e K^{i} \varsubsetneqq u(g R) P+u(g R) Q \subseteq e K^{i}+u(g R) Q$. Hence $e K^{i} \varsubsetneqq u(g R) Q \subseteq$ $e K^{i-1} Q$. It follows from Lemma 3.1 that $e K^{i-1} P=e K^{i}$. Thus $P=\operatorname{ann}_{R}\left(e K^{i-1} / e K^{i}\right)$. Now set $Q=\operatorname{ann}_{R}\left(e K^{i} / e K^{i+1}\right)$. Then $Q \neq P$ and $e K^{i}=e K^{i} P+e K^{i} Q \subseteq u(g R) P+e K^{i+1} \subseteq$ $u(g P)+u(g R) K \subseteq e K^{i}$. Therefore $u(g K)=e K^{i}$. Let $s$ be a positive integer such that $\operatorname{ann}_{R}\left(e K^{s-1} / e K^{s}\right)=P$. Then $e K^{s-1} g$ is not contained in $e K^{s}$ because $g \notin P$. The minimality of $i$ gives $s \geqslant i$. It follows from the periodicity of the sequence of ideals ann $\left(e K^{j} / e K^{j+1}\right)$ that $i \leqslant k$.

Let $f$ also be a primitive idempotent of $R$ and let $j$ and $v$ be determined by $f$ in the same way that $i$ and $u$ were determined by $e$. Set $H=\operatorname{Hom}_{R}(e R, f R)$. The aim is to identify $H$ as either $T$ or $J(T)$ according to the relative sizes of $i$ and $j$. Let $h \in H$. Because $h u(g R) \subseteq f R$, we have $h u(g R) \subseteq f K^{j-1}$ by definition of $j$. Hence $h u(g K) \subseteq f K^{j}=v(g K)$. Thus $h$ induces an element $v^{-1} h u$ of $\operatorname{End}_{R}(g K)$. We shall now investigate the function from $H$ to $T$ which sends $h$ to $v^{-1} h u$.

Case (a). Suppose that $i>j$. Let $h \in H$. Then $h\left(e K^{i-1}\right)=h(e R) K^{i-1} \subseteq f K^{i-1}$. Set $Q=\operatorname{ann}_{R}\left(f K^{i-1} / f K^{i}\right)$. We have $1 \leqslant j<i \leqslant k$. Hence $j<i<k+j$. Therefore $i \neq j \bmod (k)$, i.e. $\operatorname{ann}_{R}\left(f K^{i-1} / f K^{i}\right) \neq \operatorname{ann}_{R}\left(f K^{j-1} / f K^{j}\right)$, i.e. $Q \neq P$. Hence $P+Q=R$ and $h\left(e K^{i-1}\right)=$ $h\left(e K^{i-1} P\right)+h\left(e K^{i-1} Q\right)=h\left(e K^{i}\right)+h\left(e K^{i-1}\right) Q \subseteq f K^{i}+f K^{i-1} Q=f K^{i}$. If $i+1<k+j$, we can repeat the argument and obtain $h\left(e K^{i-1}\right) \subseteq f K^{i+1}$. After a finite number of steps, we have $h\left(e K^{i-1}\right) \subseteq f K^{k+j-1}$. Hence $h\left(e K^{i}\right) \subseteq f K^{k+j}$, i.e. $h u(g K) \subseteq v(g K) K^{k}$, i.e. $v^{-1} h u(g K) \subseteq g K^{k+1}$, i.e. $v^{-1} h u \in J(T)$. Now let $t \in J(T)$. We shall show that $t=v^{-1} h u$ for some $h \in H$. We have $t(g K) \subseteq g K^{k+1}$. Because $e K^{i}=u(g K)$, it makes sense to set 
$a(x)=v t u^{-1}(x)$ for all $x \in e K^{i}$. We have $a\left(e K^{i}\right)=v t(g K) \subseteq v\left(g K^{k+1}\right)=v(g K) K^{k}=f K^{k+j}$. Thus $a: e K^{i} \rightarrow f K^{k+j}$ is a right $R$-module homomorphism. We fix $x \in e K^{i}$ with $x \notin e K^{i+1}$. Let $w$ be the restriction of $a$ to $x R$. Because $R$ is a non-singular ring, any extension of $w$ to an element of $H$ will also be an extension of $a$. Suppose that there is a homomorphism $b: f R \rightarrow e R$ with $b w(x)=x$. We have $e K^{i+1} \subsetneq x R=b w(x R)=b a(x R) \subseteq b a\left(e K^{i}\right) \subseteq$ $b\left(f K^{k+j}\right)=b(f R) K^{k+j} \subseteq e K^{k+j}$. Hence $e K^{i+1} \sqsubseteq e K^{k+j}$, i.e. $i+1>k+j$. This is a contradiction because $k \geqslant i$ and $j \geqslant 1$. Hence there is no such $b$. Therefore $w$ can be extended to an element $h$ of $H$, by Lemma 4.1. It follows that $h$ is an extension of $a$ and that $t=v^{-1} a u=v^{-1} h u$. Thus $h \rightarrow v^{-1} h u$ gives a bijective function between $H$ and $J(T)$. We used $w$ rather than $a$ above because the domain of $a$ may not be a cyclic submodule of $e R$.

Case (b). Suppose that $i<j$. We shall identify $H$ with $T$. Let $t \in T$. As above set $a=v t u^{-1}: e K^{i} \rightarrow f K^{j}$, fix $x \in e K^{i}$ with $x \notin e K^{i+1}$, and let $w$ be the restriction of $a$ to $x R$. Suppose that there is a homomorphism $b: f R \rightarrow e R$ with $b w(x)=x$. Then $e K^{i+1} \subsetneq x R=$ $b w(x R)=b a(x R) \subseteq b\left(f K^{j}\right) \subseteq e K^{j}$. Hence $i+1>j$, which is a contradiction. Therefore no such $b$ exists, and $w$ can be extended to an element $h$ of $H$ by Lemma 4.1. We have $t=v^{-1} h u$ and we can identify $H$ with $T$ via $h \rightarrow v^{-1} h u$.

We do not wish to consider the case $i=j$.

We drop the special meanings for $e, i, u, f, j, v$.

Let $e_{1}, \ldots, e_{n}$ be orthogonal primitive idempotents of $R$ adding to 1 . For each $j$, let $i(j)$ be the smallest positive integer $s$ such that $P=\operatorname{ann}_{R}\left(e_{j} K^{s-1} / e_{j} K^{s}\right)$. From above, we have $i(j) \leqslant k$. Suppose that there is a non-negative integer $i$ with $K^{i} P=K^{i}$. Then for every primitive idempotent $e$ and every integer $j \geqslant i$ we have $K^{j} P=K^{j}$ and $P \neq$ ann $\left(e K^{j} / e K^{j+1}\right)$; this is a contradiction. Hence, for each integer $s$ with $1 \leqslant s \leqslant k$, there is an integer $j$ with $1 \leqslant j \leqslant n$ such that $e_{j} K^{s-1} P \neq e_{j} K^{s-1}$, i.e. $P=\operatorname{ann}\left(e_{j} K^{s-1} / e_{j} K^{s}\right)$, i.e. $i(j)=s$. We can arrange the numbering so that $i(1)=i(2)=\ldots=i\left(j_{k}\right)=k, i\left(j_{k}+1\right)=$ $\ldots=i\left(j_{k}+j_{k-1}\right)=k-1, \ldots, \ldots=i(n)=1$. For $1 \leqslant s, t \leqslant n$, set $H_{s t}=\operatorname{Hom}_{R}\left(e_{t} R, e_{s} R\right)$. Then $R$ is isomorphic to the ring of $n$ by $n$ matrices with $(s, t)$-entries in $H_{s t}$. If $i(s)<i(t)$ then we can identify $H_{s t}$ with $J(T)$ as in case (a). If $i(s)>i(t)$ then we can take $H_{s t}=T$ by case (b).

COROLLARY 4.3. Let $R$ be an indecomposable non-singular serial ring with finite Krull dimension. Then there is a division ring $D$ such that $R$ is isomorphic to a blocked upper triangular matrix ring in which the above-diagonal blocks are full sets of matrices over $D$, and each diagonal block is a ring as described in Theorem 4.2 which has a full matrix ring over $D$ as its quotient ring.

Proof. This follows from Theorem 4.2 and the last paragraph of Section 2 combined with [1, Theorem 6.10 and Corollary 3.3].

\section{REFERENCES}

1. A. W. Chatters and C. R. Hajarnavis, Rings in which every complement right ideal is a direct summand, Quart. J. Math. Oxford Ser. (2) 28 (1977), 61-80.

2. A. W. Chatters, A note on Noetherian orders in Artinian rings, Glasgow Math. J. 20 (1979), 125-128.

3. A. W. Chatters and C. R. Hajarnavis, Rings with chain conditions (Pitman, 1980).

4. D. Eisenbud and P. Griffith, Serial rings, J. Algebra 17 (1971), 389-400.

5. R. Gordon and J. C. Robson, Krull dimension, Mem. Amer. Math. Soc. 133 (1973). 
6. T. H. Lenagan, Reduced rank in rings with Krull dimension, Ring Theory (Proc. Antwerp Conference, 1978), Lecture Notes in Pure and Appl. Math. 51 (Dekker, 1979), 123-131.

7. L. S. Levy, Torsion-free and divisible modules over non-integral-domains, Canad. J. Math. 15 (1963), 132-151.

8. S. Singh, Serial right Noetherian rings, Canad J. Math. 36 (1984), 22-37. 319-333.

9. M. H. Upham, Serial rings with right Krull dimension one, J. Algebra 109 (1987), 187-222.

10. R. B. Warfield Jr., Serial rings and finitely presented modules, J. Algebra 37 (1975),

11. R. B. Warfield Jr., Bezout rings and serial rings, Comm. Algebra, 7 (1979), 533-545.

12. M. H. Wright, Certain uniform modules over serial rings are uniserial, Comm. Algebra 17 (1989), 441-469. 99-116.

13. M. H. Wright, Serial rings with right Krull dimension one, II, J. Algebra 117 (1988),

14. M. H. Wright, Krull dimension in serial rings, J. Algebra 124 (1989), 317-328.

School of Mathematics

UNIVERSITY OF BRISTOL

UNIVERSITY WALK

BRISTOL BS8 ITW 